\title{
openheart Ten-year follow-up of cardiac function and neural regulation in a group of amateur half-marathon runners
}

\author{
Beatrice De Maria (D) , ${ }^{1}$ Mariana de Oliveira Gois, ${ }^{2}$ Aparecida Maria Catai, ${ }^{2}$ \\ Christian Marra, ${ }^{1}$ Daniela Lucini, ${ }^{3,4}$ Alberto Porta (D) ,, ${ }^{5,6}$ Massimo Pagani, ${ }^{3}$ \\ Laura Adelaide Dalla Vecchia (i) ${ }^{1}$
}

To cite: De Maria B, de Oliveira Gois M, Catai AM, et al. Ten-year follow-up of cardiac function and neural regulation in a group of amateur half-marathon runners. Open Heart 2021;8:e001561.

doi:10.1136/

openhrt-2020-001561

Received 28 December 2020 Revised 22 January 2021 Accepted 25 January 2021

Check for updates

(c) Author(s) (or their employer(s)) 2021. Re-use permitted under CC BY-NC. No commercial re-use. See rights and permissions. Published by BMJ.

${ }^{1}$ Department of Cardiology, Istituti Clinici Scientifici Maugeri IRCCS, Milano, Italy ${ }^{2}$ Department of Physical Therapy, Federal University of São Carlos, São Carlos, Brazil ${ }^{3}$ Dipartimento di Biotecnologie Mediche e Medicina Traslazionale, University of Milan, Milano, Italy

${ }^{4}$ Exercise Medicine Unit, Humanitas Clinical and Research Center IRCCS, Rozzano, Italy

${ }^{5}$ Department of Biomedical Sciences for Health, University of Milan, Milano, Italy ${ }^{6}$ Department of Cardiothoracic Vascular Anesthesia and Intensive Care, Policlinico San Donato, San Donato Milanese, Italy

Correspondence to

Dr Laura Adelaide Dalla Vecchia laura.dallavecchia@icsmaugeri.

\section{ABSTRACT}

Objective In the last years, a debate exists about type, intensity and frequency of physical exercise that is really indicated to protect healthy subjects from cardiovascular disease. Regular physical training has been associated with an improved cardiovascular risk profile, but it has also been demonstrated that strenuous and uncontrolled physical exercise could be dangerous, in terms of increased cardiovascular morbidity and mortality. In the present study, we evaluated a group of 35 amateur half-marathon runners, who were likewise studied 10 years before (B). The results of $B$ suggested that an increased cardiac sympathetic modulation could potentially represent a negative prognostic factor. The aim of this follow-up was to assess the medium-longterm effects of moderate to vigorous physical training on the cardiovascular neural control, cardiac function and occurrence of cardiovascular diseases.

Methods Each enrolled subject underwent: (1) an interview and physical examination to ascertain the presence of cardiovascular disease; (2) standing test to evaluate the cardiovascular neural control by means of heart rate variability (HRV), arterial blood pressure (AP) variability and baroreflex sensitivity (BRS); (3) transthoracic echocardiography to evaluate cardiac function.

Results At 10-year follow-up (FU), in this group of middle-aged athletes the occurrence of cardiovascular diseases was low, not unlike that of the overall population. The results of HRV analysis showed a decreased sympathetic and increased vagal modulation directed to the heart, compared with B. In addition, HRV, AP variability and BRS indices showed a physiological response to active standing. Finally, athletes had normal echocardiographic measures.

Conclusion We conclude that in our group of athletes a regular moderate-vigorous physical training through the 10 years was quite beneficial as the prevalence of sympathetic cardiac modulation observed at B was not accompanied by increased cardiovascular risk, on the contrary a slight prevalence of vagal indices was observed at FU.

\section{INTRODUCTION}

Physical inactivity, along with smoking, poor dietary habits, overweight, high blood

\section{Key questions}

What is already known about this subject?

- The complexity of exercise induced changes on cardiac function and cardiovascular neural regulation, varying from favourable and protective to dangerous and hazardous, renders the issue still largely open. Several studies have demonstrated the presence of increased cardiac sympathetic modulation in athletes, arguing a possible detrimental prognostic role.

What does this study add?

- In the present study, a group of amateur halfmarathon runners, likewise studied 10 years before (Open Heart 2014;1:e000005), was evaluated with the aim of assessing the medium-long-term effects of moderate to vigorous physical training on the cardiovascular neural control, cardiac function and occurrence of cardiovascular disease.

- In the first study, these middle-aged athletes were characterised by increased sympathetic modulation and slight modifications of the diastolic function. In this 10-year follow-up study, the same subjects showed a low occurrence of cardiovascular diseases, not unlike that of the overall population, a decreased sympathetic and increased vagal modulation directed to the heart compared with the previous study, a physiological baroreflex sensitivity and normal echocardiographic findings. Of notice, they had continued to practice regular physical exercise but at lower intensity through the years.

How might this impact on clinical practice?

- This study suggests that a regular moderate-vigorous physical training over many years was beneficial in a group of middle-aged amateur half-marathon runners.

- These findings also provide insights about the correct type, intensity and frequency of physical exercise that is really indicated to protect healthy subjects from cardiovascular diseases.

pressure, dyslipidaemia and diabetes, has been identified as one of the modifiable cardiovascular risk factors by the European Society of Cardiology. ${ }^{1}$ Nevertheless, in 
the last years, a debate exists about type, intensity and frequency of physical exercise that is really indicated to protect healthy subjects from cardiovascular diseases and to adequately rehabilitate heart patients. ${ }^{2-9}$ From one hand regular physical training has been associated to an improved cardiovascular risk profile, ${ }^{410}{ }^{11}$ from the other hand a strenuous and uncontrolled physical training has been linked to the development of negative prognostic factors potentially favouring the onset of cardiovascular diseases. ${ }^{5}{ }^{12}$

It is well known that physical training is accompanied by a transient modification of cardiac function and cardiovascular neural control. ${ }^{5}{ }^{13-15}$ However, some of these adjustments, such as reduced heart volumes and increased sympathetic cardiac activity, may outlast the physical performance in amateur triathletes and in amateur half-marathon runners. ${ }^{5}{ }^{13}$ An increased sympathetic cardiac activity is known to be a negative prognostic index in several conditions, ${ }^{16-19}$ however, its significance in athletes is under discussion, in particular, it is unclear whether it may favour adverse cardiovascular events. ${ }^{131420}$ A longitudinal assessment of athletes could provide important insights about this open issue.

The aim of this study was to evaluate the cardiovascular neural control and cardiac function of a group of amateur half-marathon runners, who were likewise studied 10 years before within the Corripavia protocol, ${ }^{13}$ in order to provide the medium-long-term effects of moderate to vigorous physical training on the cardiovascular neural control, cardiac function and occurrence of cardiovascular diseases. The cardiovascular neural control was evaluated using the heart rate variability (HRV), arterial blood pressure (AP) variability and baroreflex sensitivity (BRS) analysis at rest and during an active standing manoeuvre, the presence of cardiovascular disease and cardiac function by history, physical examination and transthoracic echocardiography.

\section{METHODS \\ Study design}

This study is a 10-year follow-up in a group of amateur half-marathon runners who participated to the fifth Corripavia race and related protocol. ${ }^{13}$ After verifying the state of life through the digital regional systems, all participants were invited by phone and/or email to schedule an appointment at our centre (IRCCS Istituti Clinici Scientifici Maugeri, Milan, Italy) to undergo an echocardiographic examination and a standing test to evaluate the cardiac function and the cardiovascular neural control, respectively, analogously to the previous protocol. ${ }^{13}$ In the present study, the continuous recording of blood pressure was also included to allow the calculation of the BRS. All patients were interviewed to collect anamnestic, demographic and sporting habits data. For convenience, the data of the first and the present protocol will hereinafter be referred to as baseline (B) and follow-up (FU).
No a priori inclusion and exclusion criteria were applied.

\section{Echocardiographic assessment}

A standard two-dimensional echocardiographic and colour Doppler examination (Vivid E9, GE Healthcare, Little Chalfont, GB) with a $2-4.5 \mathrm{MHz}$ transducer was obtained for all subjects in the left lateral recumbent position. Imaging was acquired and stored digitally for later off-line blinded analysis (averaging three consecutive cycles for all modalities) by one experienced cardiologist and one sonographer (LADV and CM). To allow comparison, the same measurements were considered as for B. ${ }^{13}$ Measurements and calculations were obtained according to recommendations. ${ }^{21}$

\section{Cardiovascular neural regulation assessment}

Tests were performed in the morning after the echocardiographic examination, in a quiet room with comfortable temperature, after a good night's sleep. All the participants were asked to avoid caffeinated and alcoholic beverages in the 24 hours preceding the test. They were studied 72 hours far away from the last bout of physical exercise to avoid the short-term autonomic and cardiovascular confounding after-effects induced by recent training sessions. ${ }^{14}$ In addition, none of them had participated in a competition for at least 4 weeks, as for the previous study. ${ }^{13}$

ECG was obtained by modified lead II (Marazza, Monza, Italy) and beat-to-beat AP was monitored by a photoplethysmographic device (Finometer Midi, Finapress Medical System, The Netherlands). Sampling rate was $1000 \mathrm{~Hz}$. Signals were acquired for $10 \mathrm{~min}$ while supine (REST) and for $10 \mathrm{~min}$ during active standing (STAND).

From the ECG, the time elapsed between two successive R-waves, that is the RR interval (RR) was derived as the temporal distance between two consecutive $\mathrm{R}$ peaks by an automatic algorithm. Detections were manually checked to avoid misidentifications. Stationary segments of 300 consecutive beats were selected for REST and STAND. After linear detrending of the selections, mean and variance $\left(\sigma_{\mathrm{RR}}^{2}\right)$ of RR interval were calculated and expressed in $m s$ and $\mathrm{ms}^{2}$, respectively. Within each RR interval, the maximum of the AP signal was taken as systolic AP (SAP) value.

Parametric power spectral analysis was performed to assess the frequency content of the RR interval and SAP series. The series were modelised by an autoregressive model whose order was optimised by Akaike information criterion. The sum of the spectral components of the RR series occurring in the low frequency (LF, $0.03-0.15 \mathrm{~Hz}$ ) and high frequency (HF, $0.15-0.4 \mathrm{~Hz}$ ) bands were expressed in $\mathrm{ms}^{2}$ and named $\mathrm{LF}_{\mathrm{RR}}$ and $\mathrm{HF}_{\mathrm{RR}}$, respectively. Similarly, the absolute power of SAP series in LF and HF bands was obtained, expressed in $\mathrm{mmHg}^{2}$ and named $\mathrm{LF}_{\mathrm{SAP}}$ and $\mathrm{HF}_{\mathrm{SAP}}$, respectively. $\mathrm{LF}_{\mathrm{RR}}$ and $\mathrm{HF}_{\mathrm{RR}}$ were also expressed in normalised units $\left(\mathrm{LF}_{\mathrm{nu}, \mathrm{RR}}\right.$ and 
$\mathrm{HF}_{\text {nu,RR }}$, respectively). $\mathrm{HF}_{\mathrm{RR}}$ and $\mathrm{HF}_{\text {nu.RR }}$ were considered as markers of the vagal modulation directed to the sinus node, ${ }^{22}$ while $\mathrm{LF}_{\mathrm{RR}}$ and $\mathrm{LF}_{\mathrm{nu}, \mathrm{RR}}$ as indices providing some information about the sympathetic modulation directed to the sinus node. ${ }^{23}$ Combining HRV information embedded in time ( $R R$ and RR variance) and frequency domain indices (stand-rest difference in $\mathrm{LF}_{\mathrm{RR}, \mathrm{nu}}$ ) into a $\%$ ranked Autonomic Nervous System Index (ANSI) provides unitary information on individual autonomic cardiac regulation against a relatively large benchmark population. ${ }^{25}$

The BRS was estimated by the spectral method, ${ }^{15} 17$ as the square root of the ratio between $\mathrm{LF}_{\mathrm{RR}}$ and $\mathrm{LF}_{\mathrm{SAP}}$ $\left(\mathrm{BRS}_{\mathrm{LF}}\right)$ and as the square root of the ratio between $\mathrm{HF}_{\mathrm{RR}}$ and $\mathrm{HF}_{\mathrm{SAP}}\left(\mathrm{BRS}_{\mathrm{HF}}\right)$ after verification of high squared coherence. $\mathrm{BRS}_{\mathrm{LF}}$ and $\mathrm{BRS}_{\mathrm{HF}}$ were expressed in ms/ $\mathrm{mmHg}$.

\section{Statistical analysis}

Paired t-test, or the Wilcoxon signed rank test when appropriate, was applied to test the significance of the difference between echocardiographic parameters at the follow-up (ie, B vs FU) and the difference between BRS and SAP variability indices at REST and during STAND. Two-way repeated measures analysis of variance with Holm-Sidak test for multiple comparison, two factors repetition, was used to test the significance of the difference in the HRV indices between the two different periods (B and FU) during REST and STAND. All analyses were carried out using a commercial statistical programme (Sigmaplot, Systat Software, Chicago, IL, USA, V.11.0). A $\mathrm{p}<0.05$ was always considered as significant.

\section{RESULTS}

\section{Clinical follow-up}

Out of 35 non-elite athletes of the original protocol, nine did not reply to repeated calls and emails although certainly alive. Eighteen subjects (mean age $52.28 \pm 7.99$ years, range 39-65years, 17 men) agreed to the full protocol, while eight underwent only the clinical interviews, because of working $(75 \%)$ or family $(25 \%)$ reasons.

All the enrolled subjects were normal weighted (body mass index $23.06 \pm 1.72 \mathrm{~kg} / \mathrm{m}^{2}$ ) with a mean abdominal circumference of $87 \pm 6.72 \mathrm{~cm}$, (all within normal limits: $<192 \mathrm{~cm}$ for men, $<88 \mathrm{~cm}$ for women). Two of them $(8 \%)$ were light smokers, as in B. As to the eating habits, 12 participants $(46 \%)$ declared to take vitamin supplement, $3(12 \%)$ were vegetarians and $6(23 \%)$ teetotalers.

At clinical follow-up, three subjects $(12 \%)$ had developed hypertension, one paroxysmal atrial fibrillation $+30 \%$ carotid stenosis $(4 \%)$ and one $(4 \%)$ pure hypercholesterolemia (in presence of family history).

As to the sporting habits, five subjects (19\%) had stopped running for $>3$ years, of which two switched to regular walking, one to swimming and two to biking; overall these five subjects were training $4.67 \pm 1.53$ hours
Table 1 Echocardiographic parameters of amateur athletes: a 10-year follow-up

\begin{tabular}{|c|c|c|}
\hline & FU (2018) & B (2008) \\
\hline LV EDV, $\mathrm{cm}^{3}$ & $119.89 \pm 16.13$ & $114.33 \pm 22.35$ \\
\hline LV ESV, $\mathrm{cm}^{3}$ & $50.06 \pm 12.29$ & $42 \pm 8.37^{*}$ \\
\hline LV EF, \% & $60.94 \pm 4.36$ & $61.73 \pm 6.03$ \\
\hline LV mass, $\mathrm{g}$ & $172.78 \pm 34.74$ & $177.71 \pm 39.06$ \\
\hline GLS, \% & $-17.21 \pm 1.70$ & $-19.75 \pm 2.95$ \\
\hline Aortic root diameter, mm & $33.56 \pm 4.42$ & $32.76 \pm 2.97$ \\
\hline Max LA area, $\mathrm{cm}^{2}$ & $19.06 \pm 3.98$ & $14.26 \pm 2.86^{\star}$ \\
\hline Min LA area, $\mathrm{cm}^{2}$ & $10.58 \pm 3.10$ & $7.78 \pm 2.14^{\star}$ \\
\hline RVOT prox, mm & $27.22 \pm 1.59$ & $29.64 \pm 2.79$ \\
\hline RVI diameter, mm & $34.61 \pm 2.11$ & $33.24 \pm 1.89$ \\
\hline TAPSE, $\mathrm{mm}$ & $25.17 \pm 2.77$ & $23.45 \pm 1.12$ \\
\hline $\mathrm{E}, \mathrm{m} / \mathrm{s}$ & $0.56 \pm 0.13$ & $0.72 \pm 0.12^{\star}$ \\
\hline $\mathrm{A}, \mathrm{m} / \mathrm{s}$ & $0.46 \pm 0.93$ & $0.51 \pm 0.12$ \\
\hline E/A ratio & $1.23 \pm 0.25$ & $1.46 \pm 0.34$ \\
\hline DT, ms & $207 \pm 78.64$ & $201.47 \pm 36.99$ \\
\hline $\mathrm{e}^{\prime}, \mathrm{cm} / \mathrm{s}$ & $9 \pm 2$ & $11 \pm 2^{*}$ \\
\hline E/e' ratio & $6.03 \pm 1.27$ & $7.71 \pm 1.16^{*}$ \\
\hline ICV diameter, mm & $18.94 \pm 3.47$ & $19.98 \pm 1.99$ \\
\hline
\end{tabular}

${ }^{*} \mathrm{p}<0.05 \mathrm{~B}$ vs FU.

$A$, mitral A wave filling velocity; $B$, basal; DT, mitral deceleration time; E, mitral E wave filling velocity; $e^{\prime}$, early diastolic myocardial velocity; EDV, end-diastolic volume; EF, ejection fraction; ESV, end-systolic volume; FU, follow-up; GLS, global longitudinal strain; ICV, inferior cava vein; LA, left atrium; LV, left ventricular; RVOT prox, right ventricular outflow tract proximal diameter; RVI, right ventricular inflow; TAPSE, tricuspid anular plane systolic excursion.

per week. Of those still running, 12 subjects had continued to run the half-marathon distance for $6.58 \pm 3.2$ hours per week, and one had switched to the ultramarathon with $10.5 \pm 0.71$ hours of running per week. Overall, at FU, the mean training was $6.54 \pm 3.07$ hours per week compared with $14.65 \pm 2.89$ hours that was the mean time dedicated to sport weekly in B $(\mathrm{p}<0.001)$.

All the participants who underwent the complete experimental protocol were free of any sign or symptom of pre-syncope during the standing test, notably they all had a good night sleep before the tests $(6.78 \pm 0.81$ hours of sleep).

\section{Echocardiographic follow-up}

Table 1 shows the echocardiographic findings. They are compared with those acquired at $\mathrm{B}$. With respect to $\mathrm{B}$, the left ventricular (LV) end-systolic volume, Max left atrium (LA) and Min LA area significantly increased, while E, $\mathrm{e}^{\prime}$ and $\mathrm{E} / \mathrm{e}^{\prime}$ significantly decreased.

\section{HRV follow-up}

HRV data are shown in table 2. Compared with $\mathrm{B}$, at FU, athletes were characterised by an higher RR at REST, a lower $\mathrm{LF}_{\mathrm{RR}}$ and $\mathrm{LF}_{\mathrm{nu}, \mathrm{RR}}$ both at REST and during STAND 
Table 2 Heart rate variability parameters of amateur athletes: a 10-year follow-up

\begin{tabular}{|c|c|c|c|c|}
\hline & FU (2018) & & B (2008) & \\
\hline & REST & STAND & REST & STAND \\
\hline $\mathrm{RR}, \mathrm{ms}$ & $1069.67 \pm 155.87$ & $856.07 \pm 141.02^{*}$ & $930.31 \pm 194.69 \dagger$ & $765.90 \pm 192.34^{*}$ \\
\hline$\sigma_{\mathrm{RR}}^{2}, \mathrm{~ms}^{2}$ & $1727.56 \pm 717.73$ & $1811.14 \pm 957.48$ & $2711.12 \pm 1713.96$ & $2577.85 \pm 1720.54$ \\
\hline $\mathrm{LF}_{\mathrm{RR}}, \mathrm{ms}^{2}$ & $411.83 \pm 426.86$ & $638.21 \pm 791.01$ & $739.41 \pm 546.01 \dagger$ & $1053.37 \pm 939.61 \dagger$ \\
\hline $\mathrm{LF}_{\mathrm{nu}, \mathrm{RR}}, \mathrm{nu}$ & $31.76 \pm 15.16$ & $42.23 \pm 22.20^{*}$ & $53.68 \pm 24.63 \dagger$ & $78.61 \pm 18.04^{*} \dagger$ \\
\hline $\mathrm{HF}_{\mathrm{RR}}, \mathrm{ms}^{2}$ & $691.62 \pm 220.91$ & $434.79 \pm 199.77^{\star}$ & $630.43 \pm 761.49$ & $358.30 \pm 685.99^{*}$ \\
\hline $\mathrm{HF}_{\mathrm{nu}, \mathrm{RR}}, \mathrm{nu}$ & $65.29 \pm 13.04$ & $42.18 \pm 18.87^{\star}$ & $44.07 \pm 24.32 \dagger$ & $18.59 \pm 19.33^{*}$ \\
\hline
\end{tabular}

Data are presented as mean \pm SD.

${ }^{*} p<0.05$ REST vs STAND.

$\mathrm{tp}<0.05 \mathrm{~B}$ vs FU in the same experimental condition.

$\mathrm{B}$, basal; FU, follow-up; HF, high frequency; $\mathrm{HF}_{\mathrm{RR}}$, absolute power of RR in HF band; $\mathrm{HF}_{\mathrm{RR} \text { n, }}$, normalised power of RR in HF band; LF, low frequency; $L F_{R R}$, absolute power of $R R$ in $L F$ band; $L F_{R R, n u}$, normalised power of $R R$ in $L F$ band; REST, supine position; $\sigma^{2}{ }_{R R}$, $R R$ variance; $R R$, RR interval; STAND, active standing.

and a higher $\mathrm{HF}_{\mathrm{nu}, \mathrm{RR}}$ both at REST and during STAND. As in $\mathrm{B}$, also at FU, the athletes physiologically adjusted to STAND lowering the RR, increasing the $\mathrm{LF}_{n \mathrm{nu}, \mathrm{RR}}$ and decreasing the $\mathrm{HF}_{\mathrm{nu}, \mathrm{RR}}$ and $\mathrm{HF}_{\mathrm{RR}}$.

In this small population, the index ANSI was slightly increased from B to $\mathrm{FU}(56.0 \pm 28.37 .6$ vs $65.1 \pm 12.8$, $\mathrm{p}=0.20)$.

\section{SAP variability and BRS evaluation}

The results of the analysis of SAP variability and BRS are shown in table 3. Athletes had normal SAP values at REST and during STAND without any drop. Both $\sigma_{\mathrm{SAP}}^{2}$ and $\mathrm{LF}_{\mathrm{SAP}}$ physiologically increased from REST to STAND. Equally physiologically, both $\mathrm{BRS}_{\mathrm{LF}}$ and $\mathrm{BRS}_{\mathrm{HF}}$ significantly decreased during STAND. These data were not comparable with $\mathrm{B}$, as in the first study continuous AP was not included in the protocol.

\section{DISCUSSION}

In the present study, we evaluated the cardiovascular neural control and the cardiac function of a group of amateur half-marathon runners at a 10-year follow-up with the aim at evaluating the medium to long-term effects of half-marathon training. The current protocol

\begin{tabular}{|c|c|c|}
\hline & REST & STAND \\
\hline $\mathrm{SAP}, \mathrm{mmHg}$ & $130.28 \pm 11.53$ & $132.43 \pm 13.08$ \\
\hline$\sigma_{S A P^{\prime}}^{2} m_{m} g^{2}$ & $9.36 \pm 7.16$ & $15.57 \pm 9.91^{*}$ \\
\hline $\mathrm{LF}_{\mathrm{SAP}}, \mathrm{mmHg}^{2}$ & $1.37 \pm 1.16$ & $5.86 \pm 5.31^{*}$ \\
\hline $\mathrm{BRS}_{\mathrm{LP}} \mathrm{ms} / \mathrm{mmHg}$ & $21.23 \pm 8.65$ & $11.95 \pm 6.60^{*}$ \\
\hline $\mathrm{BRS}_{\mathrm{HF}} \mathrm{ms} / \mathrm{mmHg}$ & $56.85 \pm 22.65$ & $18.49 \pm 10.22^{*}$ \\
\hline
\end{tabular}

Data are presented as mean \pm SD.

${ }^{*} \mathrm{p}<0.05$ REST vs STAND.

BRS, baroreflex sensitivity; $B_{R S}$, BRS in LF band; HF, high frequency; $L F$, low frequency; $L_{S A P}$ absolute power of SAP in LF band; REST, supine position; $\sigma_{\text {SAP }}^{2}$ SAP variance; SAP, systolic arterial pressure; STAND, active standing. followed that of the fifth edition of the Corripavia halfmarathon, ${ }^{13}$ in addition blood pressure variability and arterial baroreflex sensitivity were also evaluated to better define the cardiovascular autonomic profile.

The main finding can be summarised as follows: a regular moderate-vigorous physical training maintained through 10 years was quite beneficial in this group of non-elite athletes, as (1) the prevalence of sympathetic cardiac modulation observed at B was not accompanied by increased cardiovascular risk; (2) also cardiac function did not deteriorate; (3) a more balanced cardiac sympathovagal modulation was observed at FU.

In this small, middle-aged group of half-marathon runners, the occurrence of cardiovascular disease over 10 years was low, not unlike that of the overall population. ${ }^{26}$ In the same subjects, the study conducted 10 years earlier had highlighted the presence of increased sympathetic modulation, ${ }^{13}$ arguing a possible detrimental prognostic role rather than a finalistic transient adjustment, as also raised in other studies. ${ }^{514}$ With this follow-up, we found no evidence of such worry.

In addition, the evaluation of the cardiac neural control revealed that at $\mathrm{FU}$, the athletes showed a decreased sympathetic and increased vagal modulation directed to the heart with a physiological response to active standing. In keeping, they were also characterised by a physiological resting profile and response to orthostasis in terms of SAP variability and BRS.

Finally, they had normal echocardiographic findings, although the LV systolic volume, LA dimensions, E, $\mathrm{e}^{\prime}$ and $\mathrm{E} / \mathrm{e}^{\prime}$ were consensually different from before. As underlined previously, ${ }^{13}$ these LV systolic, relaxation, compliance properties are influenced by the cardiac sympathetic control. As these athletes changed their cardiac autonomic profile, the cardiac function was consequently modified, always within normal limits. It has to be remembered that the echocardiographic parameters could also vary because of the ageing process. $^{27} 28$ 
Even with a small study population, our findings provide some detail on the controversial issue of the benefits or disadvantages of different levels of physical training through the years. This sample, although small, is of particular interest given its age and the long follow-up at 10 years. In fact, establishing whether endurance physical activity is beneficial in athletes in their $50 \mathrm{~s}$ or $60 \mathrm{~s}$ is an important issue.

Of notice, most athletes continued to practice regular physical exercise through the years, although the mean hours of training per week dropped, although still in a range of training that can be considered at least moderate.

Interestingly, at FU the athletes, in addition to a slight increase in ANSI, suggestive of improved cardiac autonomic regulation, showed a decreased sympathetic modulation directed to the heart and an enhanced vagal one, as mirrored by an higher $\mathrm{HF}_{\mathrm{RR}, \mathrm{nu}}$ and a lower $\mathrm{LF}_{\mathrm{RR}}$ and $\mathrm{LF}_{\mathrm{RR}, \mathrm{nu}}$ compared with $\mathrm{B}$. This observation is somehow opposite to what expected during the physiological ageing process, which is characterised by a progressive increase of the sympathetic tone and a decrease of the vagal one. ${ }^{29-31}$ This apparent discrepancy could just reflect the effect of the reduced amount of physical exercise weekly in a group of athletes used to train much more and to have high cardiac sympathetic modulation at rest. If so, a regular and strenuous endurance exercise practiced at the time of the first study ${ }^{11}$ likely elicited a transient increase of cardiac sympathetic drive ${ }^{32}$ that is, reversible by the modification of training intensity, that did not represent a risk factor for the development of echocardiographic abnormalities or cardiovascular disease.

The cardiovascular autonomic regulation seems to be also well preserved, as demonstrated by the normal to elevated BRS gain at rest and normal adaptation to the orthostatic challenge. Indeed, in response to the active standing, the heart rate increased, the indices of cardiac sympathetic modulation increased $\left(\mathrm{LF}_{\mathrm{RR}}\right.$ and $\left.\mathrm{LF}_{\mathrm{nu}, \mathrm{RR}}\right)$ together with the withdrawal of the indices of cardiac vagal modulation $\left(\mathrm{HF}_{\mathrm{RR}}\right.$ and $\left.\mathrm{HF}_{\mathrm{nu}, \mathrm{RR}}\right)$. The indices linked to the sympathetic modulation directed to the vessels $\left(\sigma_{\text {SAP }}^{2}\right.$ and $\left.L_{\mathrm{SAP}}\right)$ also increased, and the BRS indices decreased, as expected in healthy subjects. ${ }^{22} 24$

In conclusion, this study suggests that a regular moderate-vigorous physical training through the years was beneficial in a group of middle-aged amateur halfmarathon runners.

In fact, the slight rightward shift of normalised RR variability power, as evidenced by the higher $\mathrm{HF}_{\mathrm{RR}, \mathrm{nu}}$ at $\mathrm{FU}$, combined with the elevated BRS gain, is likely to reflect the autonomic remodelling mediated by continued endurance training, although maintained at a lower intensity, that these athletes sustained through at least 10 years.

The main strength of this study, even with a small study population, is the assessment of the long-term effect of physical training in a group of middle-aged subjects, a crucial age for the development of cardiac disease. The high percentage of dropouts and the limited sample size, together with the lack of a control group of sedentary subjects, represent a limitation of the present study.

Future research should deepen the long-term effect of moderate physical training in a larger sample of subjects, including those with sedentary habits, in order to also recognise the effects of physical training on the ageing process and the favourable effects in slowing cardiovascular function decline. ${ }^{33}$ In addition, future studies are advocated to assess the sex differences both in trained and sedentary subjects. ${ }^{9}$

Acknowledgements The authors thank all the volunteers who adhered to the experimental protocol. They thank Franco Corona, President of the Asd Atletica Cento Torri Pavia for the organisational support.

Contributors All authors have taken due care to ensure the integrity of the work, meet authorship criteria of the uniform requirements as follows. LADV and BDM contributed to conception and design of the study. LADV and MOG contributed to clinical assessment and follow-up. MOG, CM and BDM contributed to execution of tests and acquisition of data. BDM, LADV, DL, AMC and MP contributed to analysis and interpretation of the data. LADV and BDM contributed to drafting of the manuscript. All authors contributed to review and approval of the manuscript.

Funding The authors have not declared a specific grant for this research from any funding agency in the public, commercial or not-for-profit sectors.

Competing interests None declared.

Patient consent for publication Not required.

Ethics approval The study conformed to the Principles of the Declaration of Helsinki. The protocol was approved by the local Ethics Committee (ICS Maugeri Ethics Committee, Pavia, Italy, approval \# 2277CE). Each subjects signed a written informed consent.

Provenance and peer review Not commissioned; externally peer reviewed.

Data availability statement Data are available upon request to the corresponding author. All data are stored in a dedicated archive at IRCCS Istituti Clinici Scientifici Maugeri, Milan, Italy.

Open access This is an open access article distributed in accordance with the Creative Commons Attribution Non Commercial (CC BY-NC 4.0) license, which permits others to distribute, remix, adapt, build upon this work non-commercially, and license their derivative works on different terms, provided the original work is properly cited, appropriate credit is given, any changes made indicated, and the use is non-commercial. See: http://creativecommons.org/licenses/by-nc/4.0/.

\section{ORCID iDs}

Beatrice De Maria http://orcid.org/0000-0001-5360-3137

Alberto Porta http://orcid.org/0000-0002-6720-9824

Laura Adelaide Dalla Vecchia http://orcid.org/0000-0002-6516-0959

\section{REFERENCES}

1 Pelliccia A, Sharma S, Gati S. ESC guidelines on sports cardiology and exercise in patients with cardiovascular disease. Eur Heart $J$ 2020;2020.

2 Eijsvogels TMH, Thompson PD, Franklin BA. The "extreme exercise hypothesis": Recent findings and cardiovascular health implications. Curr Treat Options Cardiovasc Med 2018;20:84.

3 Schmied C. 'Paracelcus' rediscovered: searching for the right dose of physical training. Open Heart 2014;1:e000027-13.

4 Tesema G, George M, Hadgu A, et al. Does chronic high-intensity endurance training have an effect on cardiovascular markers of active populations and athletes? systematic review and metaanalysis. BMJ Open 2019;9:e032832-2019.

5 Dalla Vecchia LA, Barbic F, De Maria B, et al. Can strenuous exercise harm the heart? insights from a study of cardiovascular neural regulation in amateur triathletes. PLoS One 2019;14:e0216567.

6 Galiuto L, Fedele E, Vitale E, et al. Personalized exercise prescription for heart patients. Curr Sports Med Rep 2019;18:380-1.

7 Pedisic Z, Shrestha N, Kovalchik S, et al. Is running associated with a lower risk of all-cause, cardiovascular and cancer mortality, and is the more the better? A systematic review and meta-analysis. $\mathrm{Br} \mathrm{J}$ Sports Med 2020;54:898-905. 
8 Schnohr P, O'Keefe JH, Marott JL, et al. Dose of jogging and longterm mortality: the Copenhagen City heart study. J Am Coll Cardiol 2015;65:411-9.

9 Knechtle B, Nikolaidis PT. Sex- and age-related differences in half-marathon performance and competitiveness in the world's largest half-marathon - the GöteborgsVarvet. Res Sports Med 2018;26:75-85.

10 Shiroma EJ, Lee I-M. Physical activity and cardiovascular health: lessons learned from epidemiological studies across age, gender, and race/ethnicity. Circulation 2010;122:743-52.

11 Wen CP, Wai JPM, Tsai MK, et al. Minimum amount of physical activity for reduced mortality and extended life expectancy: a prospective cohort study. Lancet 2011;378:1244-53.

12 Croft AM, Palmer JV. Exercise and life expectancy. Lancet 2012;379:800.

13 Dalla Vecchia L, Traversi E, Porta A, et al. On site assessment of cardiac function and neural regulation in amateur half marathon runners. Open Heart 2014;1:e000005-2013.

14 Furlan R, Piazza S, Dell'Orto S, et al. Early and late effects of exercise and athletic training on neural mechanisms controlling heart rate. Cardiovasc Res 1993;27:482-8.

15 Pagani M, Somers V, Furlan R, et al. Changes in autonomic regulation induced by physical training in mild hypertension. Hypertension 1988;12:600-10.

16 La Rovere MT, Bigger JT, Marcus FI, et al. Baroreflex sensitivity and heart-rate variability in prediction of total cardiac mortality after myocardial infarction. ATRAMI (autonomic tone and reflexes after myocardial infarction) Investigators. Lancet 1998;351:478-84.

17 Pinna GD, Porta A, Maestri R, et al. Different estimation methods of spontaneous baroreflex sensitivity have different predictive value in heart failure patients. J Hypertens 2017;35:1666-75.

18 Thayer JF, Yamamoto SS, Brosschot JF. The relationship of autonomic imbalance, heart rate variability and cardiovascular disease risk factors. Int J Cardiol 2010;141:122-31.

19 Liao D, Cai J, Barnes RW, et al. Association of cardiac autonomic function and the development of hypertension: the ARIC study. Am J Hypertens 1996;9:1147-56.

20 La Rovere MT, Pinna GD, Hohnloser SH, et al. Baroreflex sensitivity and heart rate variability in the identification of patients at risk for life-threatening arrhythmias: implications for clinical trials. Circulation 2001;103:2072-7.

21 Lang RM, Badano LP, Mor-Avi V, et al. Recommendations for cardiac chamber quantification by echocardiography in adults: an update from the American Society of echocardiography and the European association of cardiovascular imaging. Eur Heart $J$ Cardiovasc Imaging 2015;16:233-71.

22 Malliani A, Pagani M, Lombardi F, et al. Cardiovascular neural regulation explored in the frequency domain. Circulation 1991;84:482-92.

23 De Maria B, Bari V, Sgoifo A, et al. Concomitant evaluation of heart period and QT interval variability spectral markers to typify cardiac control in humans and rats. Front Physiol 2019;10:1478.

24 Pagani M, Lombardi F, Guzzetti S, et al. Power spectral analysis of heart rate and arterial pressure variabilities as a marker of sympatho-vagal interaction in man and conscious dog. Circ Res 1986;59:178-93.

25 Sala R, Malacarne M, Solaro N, et al. A composite autonomic index as unitary metric for heart rate variability: a proof of concept. Eur $J$ Clin Invest 2017;47:241-9.

26 Giampaoli S, Palmieri L, Donfrancesco C, et al. Cardiovascular health in Italy. ten-year surveillance of cardiovascular diseases and risk factors: Osservatorio Epidemiologico Cardiovascolare/Health examination survey 1998-2012. Eur J Prev Cardiol 2015;22:9-37.

27 Arbab-Zadeh A, Dijk E, Prasad A, et al. Effect of aging and physical activity on left ventricular compliance. Circulation 2004;110:1799-805.

28 Beaumont A, Grace F, Richards J, et al. Left ventricular speckle tracking-derived cardiac strain and cardiac twist mechanics in athletes: a systematic review and meta-analysis of controlled studies. Sports Med 2017;47:1145-70.

29 De Maria B, Bari V, Cairo B, et al. Cardiac baroreflex hysteresis is one of the determinants of the heart period variability asymmetry. Am J Physiol Regul Integr Comp Physiol 2019;317:R539-51.

30 Milan-Mattos JC, Porta A, Perseguini NM, et al. Influence of age and gender on the phase and strength of the relation between heart period and systolic blood pressure spontaneous fluctuations. J Appl Physiol 2018;124:791-804.

31 Porta A, Bari V, De Maria B, et al. Assessing the evolution of redundancy/synergy of spontaneous variability regulation with age. Physiol Meas 2017;38:940-58.

32 lellamo F, Legramante JM, Pigozzi F, et al. Conversion from vagal to sympathetic predominance with strenuous training in highperformance world class athletes. Circulation 2002;105:2719-24.

33 Jakovljevic DG. Physical activity and cardiovascular aging: physiological and molecular insights. Exp Gerontol 2018;109:67-74.

34 Lakatta EG. Cardiovascular regulatory mechanisms in advanced age. Physiol Rev 1993;73:413-67. 